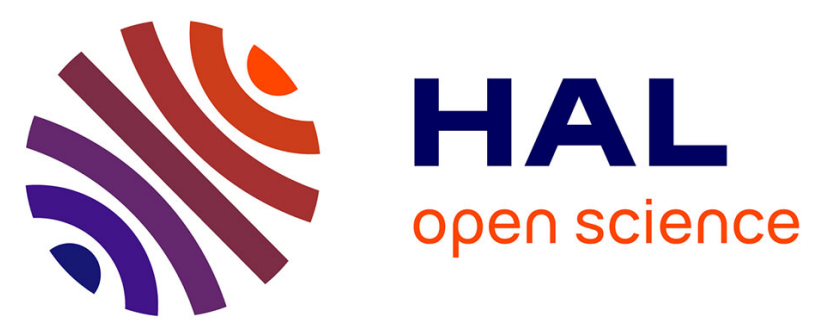

\title{
Performance analysis of the adaptive secondary mirror for the UH2.2 telescope
}

Stefan Kuiper, Wouter A Jonker, Matthew Maniscalco, Emiel van Der Ven, Robbert Voorhoeve, Mark R Chun, Olivier Lai, Jessica R Lu

\section{To cite this version:}

Stefan Kuiper, Wouter A Jonker, Matthew Maniscalco, Emiel van Der Ven, Robbert Voorhoeve, et al.. Performance analysis of the adaptive secondary mirror for the UH2.2 telescope. SPIE Astronomical Telescopes + Instrumentation, Dec 2020, Online Only, France. pp.229, 10.1117/12.2562461 . hal03371097

\section{HAL Id: hal-03371097 https://hal.science/hal-03371097}

Submitted on 28 Oct 2021

HAL is a multi-disciplinary open access archive for the deposit and dissemination of scientific research documents, whether they are published or not. The documents may come from teaching and research institutions in France or abroad, or from public or private research centers.
L'archive ouverte pluridisciplinaire HAL, est destinée au dépôt et à la diffusion de documents scientifiques de niveau recherche, publiés ou non, émanant des établissements d'enseignement et de recherche français ou étrangers, des laboratoires publics ou privés. 


\title{
Performance analysis of the adaptive secondary mirror for the UH2.2 telescope
}

\author{
S.Kuiper ${ }^{\mathrm{a}}$, W.A.Jonker ${ }^{\mathrm{a}}$, M. Maniscalco ${ }^{\mathrm{a}}$, E. van de Ven ${ }^{\mathrm{a}}$, Robbert Voorhoeve ${ }^{\mathrm{a}}$, \\ M.Chun ${ }^{\mathrm{b}}$, O. Laic, J Lu ${ }^{\mathrm{d}}$. \\ ${ }^{a}$ TNO Space\&Scientific Instrumentation, Delft, the Netherlands; ${ }^{b}$ University of Hawaii, Institute for \\ Astronomy, Hilo, USA; ${ }^{\mathrm{c}}$ Observatoire Cote d'Azur, Nice France, ${ }^{\mathrm{d}}$ University of California - \\ Berkeley, USA
}

\begin{abstract}
TNO and industrial partners are developing a new type of adaptive secondary mirrors (ASM) for the University of Hawaii 2.2-meter telescope, consisting of 210 actuators, in an overall volume of $\varnothing 63 \mathrm{~cm}$ by $15 \mathrm{~cm}$ height, and having an aspherical convex mirror-shell of $3,5 \mathrm{~mm}$ thickness. The novel actuator technology enables a compact system without active cooling that can be retro-fitted within the same mass and volume of an existing passive secondary mirror. This development enables affordable and reliable ASM systems for the world's larger telescopes as well as the many telescopes in the 2-4 meter class. This paper presents the overall design of this ASM and focusses on the performance analysis regarding its figure quality, its dynamical behavior and the related closed loop performances.
\end{abstract}

Keywords: Adaptive Secondary Mirror, Adaptive Optics

\section{INTRODUCTION}

Several observatories are exploring the use of Adaptive Secondary Mirrors (ASM) to enable effective aberration correction over wide fields of view via Ground Layer Adaptive Optics (GLAO). Such ASM's are highly complex systems due to the size, high number of actuators (hundreds to thousands), and the (aspherical) convex or concave optical surfaces. Furthermore, as these ASM's are an integral part of the telescope located atop of the spider structure, they need to be highly reliable, compact and able to cope with exposure to the changing environment.

TNO and industrial partners VDL ETG and Hyperion are developing a new type of Adaptive Secondary Mirror (ASM), based on an in-house developed variable reluctance actuator [1,3]. These actuators have a high efficiency, enabling a relatively compact overall system without active cooling that can be retro-fitted within the same mass and volume of existing passive secondary mirrors (M2). Due to the overall low complexity of this technology it is inherently reliable, which is vital to guarantee the required uptime of the telescope.

As a first project to demonstrate this technology in an observatory setting, an ASM is being developed for the University of Hawaii's 2.2-meter (UH2.2) telescope in close collaboration with the University of Hawaii. The overall goal of this project is to demonstrate this ASM technology for GLAO on Mauna Kea. The UH2.2 telescope is considered an almost ideal test-site for this new technology, given its moderate telescope size, its location close to other big telescopes, and the fact that it already has substantial AO-infrastructure into which this ASM can be integrated. Furthermore, the UH2.2 has a rotatable spider structure with two secondary mirrors, which allows the ASM to be installed on one of the positions (replacing the obsolete Coudé-focus secondary) while maintaining the availability of the original passive secondary mirror. It is expected that once successful, this development will open up new opportunities for affordable and reliable ASM's for the world's larger telescopes, as well as many telescopes in the 2-4-meter class. This paper focusses on the performance analysis that has been performed during the critical design phase of the ASM development, with regard to the wavefront error performance, the dynamical performance, and the impact of an actuator failure. For more in-depth discussion about the mechanical design and MAI-status the reader is referred to [2], while [4] discusses the test-strategy and test-preparations for this ASM. 


\section{ASM FINAL DESIGN}

Figure 1 shows a CAD rendering of the final design of this novel ASM as mounted on the rotatable spider structure of the UH2.2 telescope. This ASM contains 210 actuators in a circular grid with a radial spacing of 39mm. These actuators are based on the variable reluctance principle and have a high efficiency in terms of force per unit volume, and force per unit power. The overall volume of the ASM is $\varnothing 63 \times 13,5 \mathrm{~cm}$ which fits within the volume of the original passive M2. The overall mass of the ASM is $55 \mathrm{~kg}$, which is around $15 \mathrm{~kg}$ lower than the original passive M2.

Figure 2 shows a cross-sectional view of this ASM, with the actuators fitted at the bottom of a support structure and mechanical struts protruding through the support structure which transmit the actuator forces to the face sheet (or thin mirror shell). This mirror shell as a mechanical diameter of $\varnothing 62 \mathrm{~cm}$, and a thickness of $3,5 \mathrm{~mm}$. These actuators provide $\pm 8 \mathrm{~N}$ within its linear range $(99.5 \%)$, which via an internal mechanical lever gets amplified to $\pm 22 \mathrm{~N}$ on the strut that interfaces with the face sheet. Given the stiffness of the actuators and the facesheet this enables a linear displacement range of around $35 \mu \mathrm{m}$ PV when all the actuators are moving together, and $4 \mu \mathrm{m}$ PV inter-actuator. Recent test results on these actuators on an earlier build-standard DM are discussed in [3].

Next to these actuators' provisions are made for capacitive sensors to measure the relative displacement of the face sheet with respect to the support frame during operation. These capacitive sensors are foreseen to be used in early stages of the ASM commissioning to verify the overall actuator performances. The actuators are driven by high efficiency PulseWidth-Modulated (PWM) current amplifiers that are stowed in a $15 \times 15 \times 50 \mathrm{~cm}$ electronics box in the shadow of the ASM.

Due to the high efficiency of the actuators, the power dissipation at the ASM itself is estimated to be below 3 Watts, while the electronics drivers will dissipate around 20Watts. This low overall power dissipation allows the ASM to be operated without additional cooling which significantly simplifies the overall system and adds to the robustness.

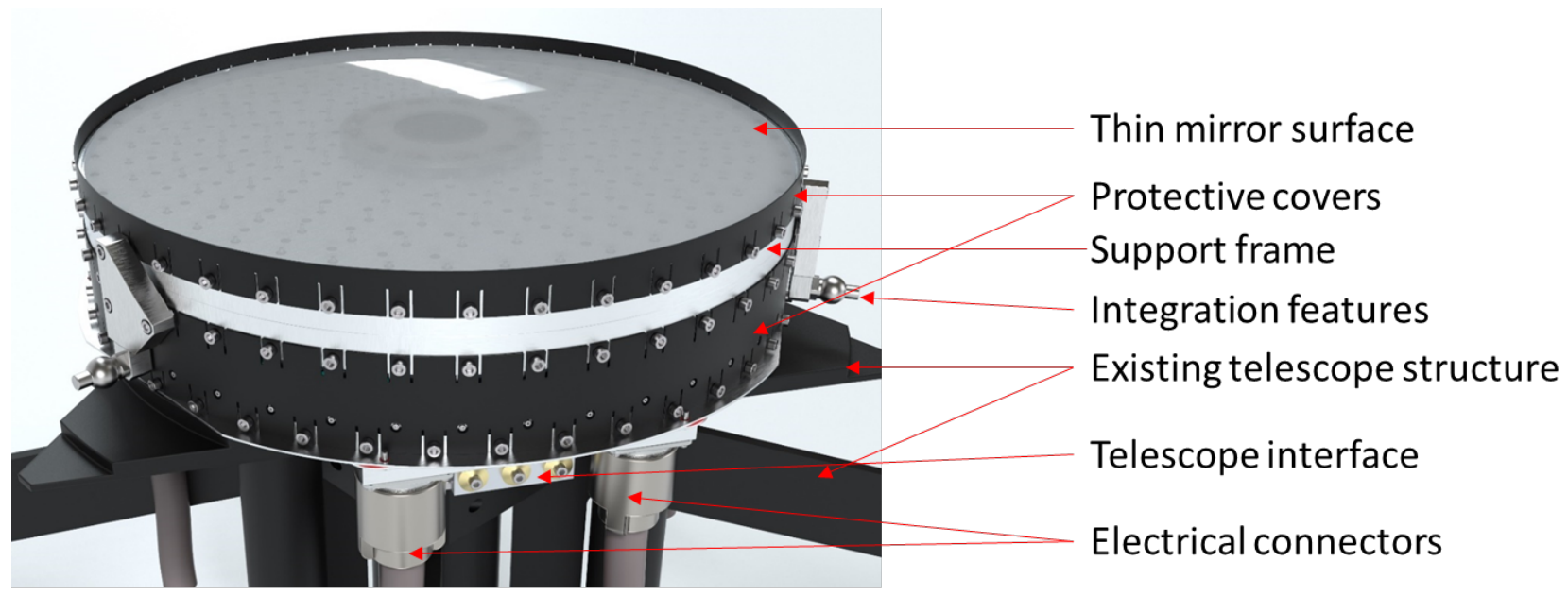

Figure 1: CAD rendering of the ASM mounted on the rotatable telescope spider structure. 


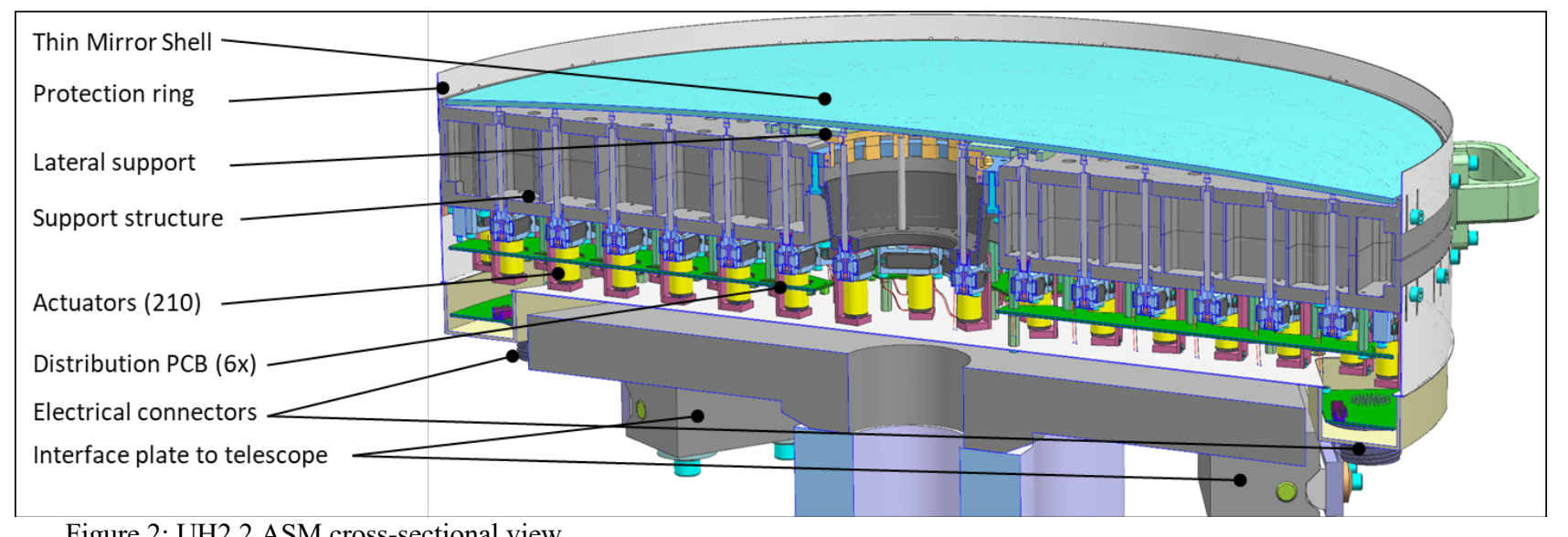

Figure 2: UH2.2 ASM cross-sectional view

Table 1: UH 2.2 ASM main features

\begin{tabular}{|l|l|l|}
\hline Basic Properties & Value & \multicolumn{2}{l|}{} \\
\hline Actuator count & 210, spacing $39 \mathrm{~mm}$ on a circular grid \\
\hline Facesheet & $\varnothing 620 \mathrm{~mm}, 3.5 \mathrm{~mm}$ thickness, convex aspherical (4.2m RoC, conic constant -3.604$)$ \\
\hline Optical Coating & protected aluminum & \multicolumn{2}{l|}{} \\
\hline Total Mass & $\sim 55 \mathrm{~kg}$ (current passive M2: $\sim 72 \mathrm{~kg})$ & Comment \\
\hline Performance Specifications & Value & \\
\hline Actuator stroke (free) & $35 \mu \mathrm{m}$ & Tunable via actuator and facesheet stiffness \\
\hline Actuator stroke (inter-actuator) & $4 \mu \mathrm{m}$ & Measured in existing prototype mirrors \\
\hline Linearity & $99.5 \%$ & 16 -bit drive electronics current resolution \\
\hline Resolution (surface) & $0.5 \mathrm{~nm}$ & Tunable via actuator and face sheet stiffness \\
\hline Actuator coupling with neighbors & $35 \%$ & Total over 210 actuators \\
\hline Actuator dissipation for flattening & $<3 \mathrm{Watts}$ & \\
\hline Drive electronics power dissipation & $<20 \mathrm{Watts}$ & Tunable via actuator stiffness \\
\hline First actuator resonance & $\sim 1 \mathrm{kHz}$ & factor $\sim 10$ below actuator resonance mode \\
\hline Achievable correction bandwidth & $\sim 100 \mathrm{to} 150 \mathrm{~Hz}$ & \\
\hline
\end{tabular}

\section{SURFACE FORM ERROR PERFORMANCE ANALYSIS}

The main performance specification of the ASM is the achievable wave front correction performance. In full Adaptive Optics (AO) operation the 'best-flat' performance target is set to $\lambda / 15$ Wave Front Error (WFE @630nm), which corresponds to $\sim 21 \mathrm{~nm}$ rms Surface Form Error (SFE). This performance needs to be achieved over the entire operational temperature range from $+20^{\circ} \mathrm{C}$ down to $-10^{\circ} \mathrm{C}$, and with a changing gravity vector when the whole telescope is moving in elevation. Besides the full AO-mode, it is also important that the ASM can be operated in a 'Static-mode' where it maintains a static figure with reasonable accuracy. This is needed during first initialization, where the initial aberrations must fall well within the detection range of the wave-front sensor. Furthermore, the ASM is also required to maintain a high-quality static figure during seeing-limited observations without high-bandwidth AO-corrections. For such static mode operation, a target WFE requirement is set at $\lambda / 4$ (corresponding to $80 \mathrm{~nm}$ rms SFE) over its entire operating range. To achieve this the ASM will be flattened with pre-calibrated 'flattening-commands' to compensate for the initial shape errors of the mirror. Furthermore, a look-up table will be used to compensate the SFE-induced by the gravity sag at the different elevation angles and at different temperature ranges. To further improve the figure accuracy for seeing-limited observations, it is foreseen that slow wavefront corrections are applied via low-order wavefront sensors. These slow figure corrections will particularly target the characteristic mode shapes corresponding to the gravity sag and thermal elastic behavior of the structure. 
The UH2.2 telescope is equipped with a refocusing stage that allows correction for focus variations during observations. As a result, spherical $\left(\mathrm{r}^{2}\right)$ aberrations of the ASM induced by for instance the thermal behavior can be compensated by this refocusing stage. This significantly reduces the stroke requirements for the ASM actuators and enables the use of a support structure with a relatively high coefficient of thermal expansion (CTE).

Table 2 shows the SFE budget of the ASM, where the SFE errors are subdivided in (i) focus aberrations which can be compensated by the refocusing stage, (ii) low and mid-spatial frequency aberrations that can be compensated by the ASM actuators, and (iii) high spatial frequency aberrations that cannot be compensated by the ASM actuators. The blue column lists the low/mid spatial frequency errors before compensation, while the orange column lists the same contributors when compensated with pre-calibrated actuator commands with an accuracy of $90 \%$. This $90 \%$ is considered a conservative assumption given the low hysteresis of the actuators $(\sim 2 \%)$. The bottom rows list the total SFE in the different modes of operation (i) initial errors, (ii) after pre-calibrated flattening, and (iii) in full AO-mode. The main figure error source is expected to be the initial figure error stemming from the manufacturing of the face-sheet. The values presented in the row named 'Initial figure errors' of Table 2 are the target specifications for manufacturing of the face sheet as agreed upon with the manufacturer. It is expected that for future ASM's higher polishing qualities can be achieved at the expense of higher polishing efforts.

The thermal behavior of the ASM structure is extensively analyzed via Finite Element Modeling (FEM), as shown in Figure 3. As can be seen in Figure 3, the kinematic strut interface between the actuators and the face sheet ensure that the face sheet can thermally 'breathe' with respect to the support frame, ensuring minimal stresses in the face sheet. As a result, the overall temperature variation yields mostly a spherical aberration of $3450 \mathrm{~nm}$ PV which can be well compensated by the refocusing stage of the telescope. The remaining aberrations are around $78 \mathrm{~nm}$ rms in the correctable range and $3 \mathrm{~nm}$ in the uncorrectable range at the maximum temperature difference of $30^{\circ} \mathrm{C}$. Notice that this is really considered worst case and better performances are expected during nominal conditions.

Another SFE- contributor is the gravity sag of the ASM structure, which has been analyzed with FEM in the two extreme cases: (i) with the gravity vector perpendicular to the mirror surface, and (ii) parallel to the mirror surface, as shown in Figure 4. Notice that a global, low spatial-frequency effect can be distinguished in which the entire support structure shows a sag with respect to its telescope interface, and a more local high spatial frequency effect of the face sheet sagging in between its actuator support points. The latter is not compensable by the actuators and is considered the main driver for the $3.5 \mathrm{~mm}$ thickness of the face sheet yielding less than $1 \mathrm{~nm}$ rms sag in the when the ASM is in the horizontal position. It is foreseen that in seeing-limited mode of operation the low spatial frequency aberrations induced by gravitysag will be compensated to a large extent via a calibrated look-up table which stores the compensation commands for the different elevation positions.

To summarize the total SFE error performance; the second-to-last row of Table 2 shows an expected SFE performance of $72 \mathrm{~nm}$ rms in static operation, and $21 \mathrm{~nm} \mathrm{rms}$ in full AO-mode which are both within the target requirements. The latter is dominated by the polishing quality of the face sheet which, if needed, can be improved by additional polishing steps. As mentioned above, it is expected that in seeing-limited mode the static figure can be further improved by (low spatial and temporal frequency) wave-front-sensors targeted at the characteristic deformation modes.

Table 2: ASM surface form error budget

\begin{tabular}{|c|c|c|c|c|c|c|}
\hline \multicolumn{7}{|c|}{ Surface form error budget (nm) } \\
\hline & Focus & \multicolumn{3}{|c|}{ Low spatial frequencies SFE (correctable) } & \multirow{3}{*}{$\begin{array}{c}\text { High spatial SFE } \\
\text { (uncorrectable) } \\
\text { RMS }\end{array}$} & \multirow{3}{*}{$\begin{array}{l}\text { Totals } \\
\text { (RSS) }\end{array}$} \\
\hline & & \multicolumn{2}{|c|}{ Initial (uncorrected) } & $\begin{array}{c}\text { Calibrated } \\
\text { correction }(90 \%)\end{array}$ & & \\
\hline & PV & PV & RMS & RMS & & \\
\hline Initial figure error & 3000 & 6000 & 630 & 63 & 20 & \\
\hline Thermal effect at $\mathrm{dT}=30^{\circ} \mathrm{C}$ & 3450 & 490 & 78 & 8 & 3 & \\
\hline Gravity effect & 631 & 1230 & 280 & 28 & 3 & \\
\hline \multicolumn{3}{|c|}{ Unpowered - target $633 \mathrm{~nm}$ SFE ( $2 \lambda$ WFE) } & 689 & & 21 & 690 \\
\hline \multicolumn{3}{|c|}{ Static mode - target $80 \mathrm{~nm}$ SFE $(K / 4 \mathrm{WFE})$} & & 69 & 21 & 72 \\
\hline \multicolumn{5}{|c|}{ AO mode - target $21 \mathrm{~nm}$ SFE $(K / 15 \mathrm{WFE})$} & 21 & 21 \\
\hline
\end{tabular}



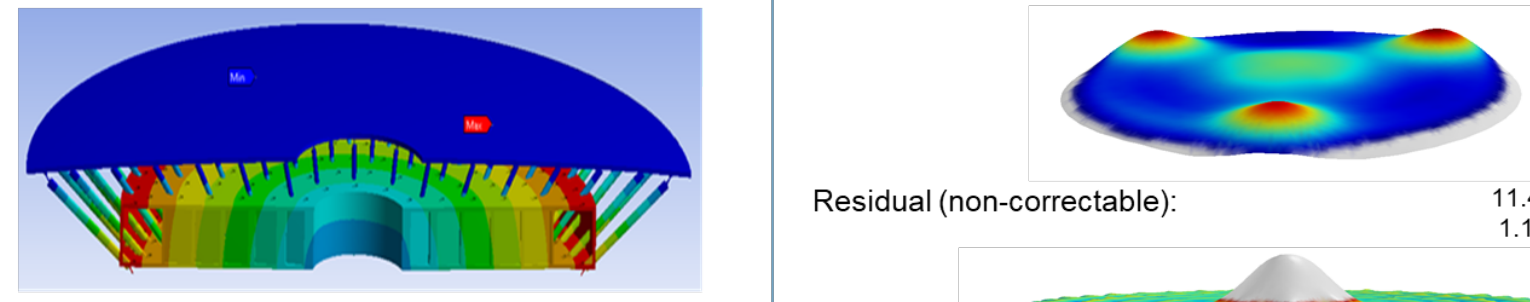

Residual (non-correctable):

$11.4 \mathrm{~nm}$ PV

$1.1 \mathrm{~nm}$ RMS

Figure 3: Thermomechanical analysis results

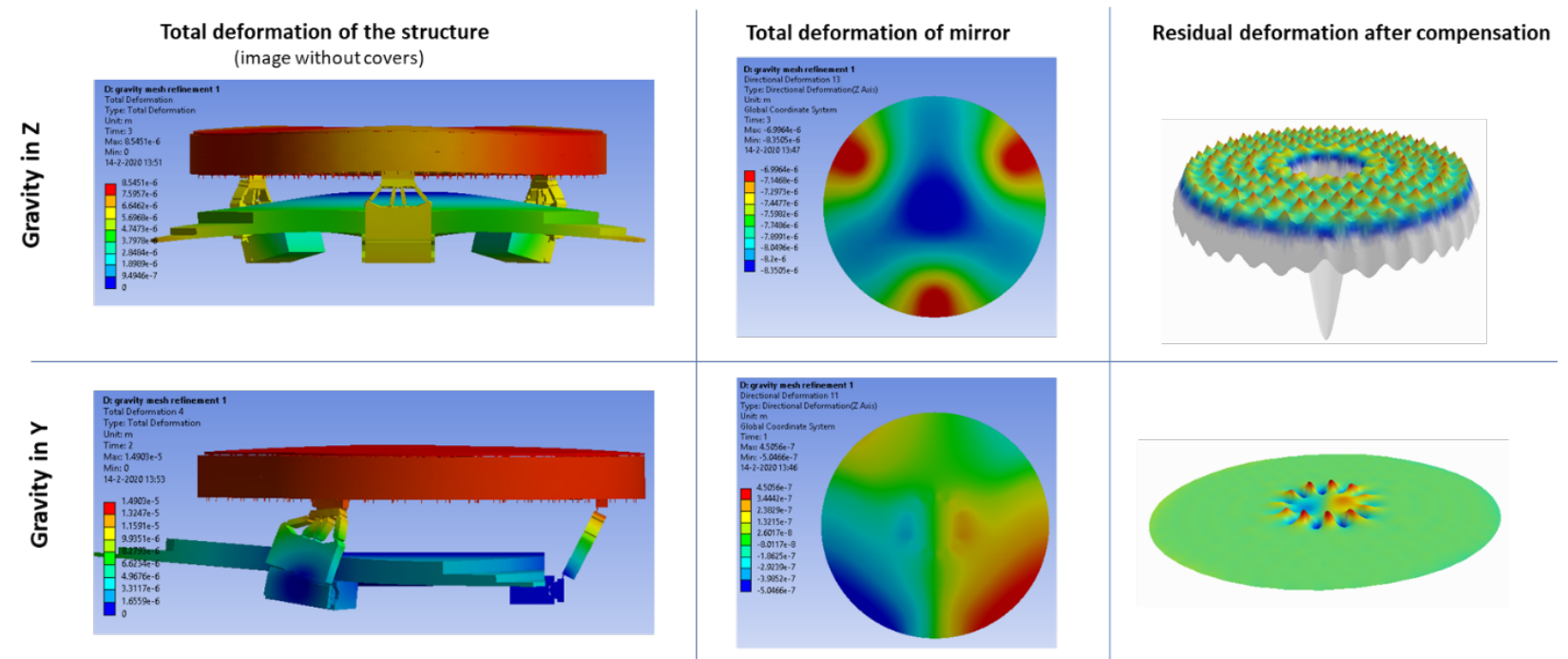

Figure 4: Effect of gravity sag on the total mirror deformations for two load cases: Gravity along the Z-axis perpendicular to the mirror surface (top) and along the Y-axis parallel to the mirror surface (bottom)

\subsection{Print-through analysis}

Print-through resulting from the bond-connections of the actuators to the face sheet may lead to specific diffraction effects due to its regular pattern. Based on optical analysis, a target budget of $10 \mathrm{~nm} \mathrm{rms}$ is defined for this pattern. Various sources of print-through are identified and analyzed with FEM. Table 3 summarizes these results in an overall print-through budget, adding up to a worst case of $6.9 \mathrm{~nm} \mathrm{rms}$ at $\mathrm{dT}=30^{\circ} \mathrm{C}$ and with gravity perpendicular to the surface which is well within the requirement. Note that for future systems print-through can be reduced by better thermal matching of the CTE at the local bonds and by CTE matching between the support structure and the mirror material.

Table 3: print-through budget

\begin{tabular}{|l|c|l|}
\hline Error source & SFE (rms) & Comments \\
\hline 1. Gravity sag in Z-axis & 1 & FEM analysis \\
\hline 2. Bond shrinkage due to epoxy curing & 0.25 & Breadboard testing \\
\hline 3. Bond CTE mismatch $\left(\mathrm{dT}=30^{\circ} \mathrm{C}\right)$ & 2.4 & FEM analysis \\
\hline 4. Strut bending due to global thermal deformation $\left(\mathrm{dT}=30^{\circ} \mathrm{C}\right)$ & 3.25 & FEM analysis \\
\hline Total & $\mathbf{6 . 9}$ & Requirement 10nm rms \\
\hline
\end{tabular}




\subsection{Impact of actuator failure}

While the actuators are inherently reliable given their low complexity and absence of wear and aging, it is of interest to understand the impact of an actuator failure on the wavefront correction performance. This impact is highly dependent on the applied actuator commands. The largest source of SFE is the initial static figure error of the face sheet (see Table 2) targeted at $5 \mu \mathrm{m}$ PV and mostly within the lower spatial frequency range. The impact of failed actuators is analyzed by generating 250 different sample shapes via a superposition of the first 21 Zernike polynomials, normalized such that the $\mathrm{PV}$ in the optical aperture is $5 \mu \mathrm{m}$. Given these sample shapes, the achievable SFE is analyzed with up to 4 randomly chosen 'failed' actuators. This analysis is done by fitting and subtracting the actuator influence functions which are generated based on FEM. The results are summarized in Figure 5. For the 250 different realizations, the top-left graph shows how often the SFE falls in a certain RMS range, with up to four randomly chosen failed actuators. The table on the bottom of Figure 5 summarizes these results, showing an average SFE of $8,77 \mathrm{~nm}$ rms with four failed actuators.

It is concluded that the SFE performance degrades gracefully with an increased number of failed actuators and the average degradation with four failed actuators is relatively small with respect to the overall SFE budget. This graceful failure is due to the relatively actuator coupling of around $35 \%$, which allow neighboring actuators to partially compensate for a failure.

Notice that the actuator design enables replacing each individual actuator during a daytime servicing operation. This is done by disconnecting the bond between the actuator and the strut via a dedicated removal tool, after this a new actuator can be placed. This procedure is further explained and shown in [2].
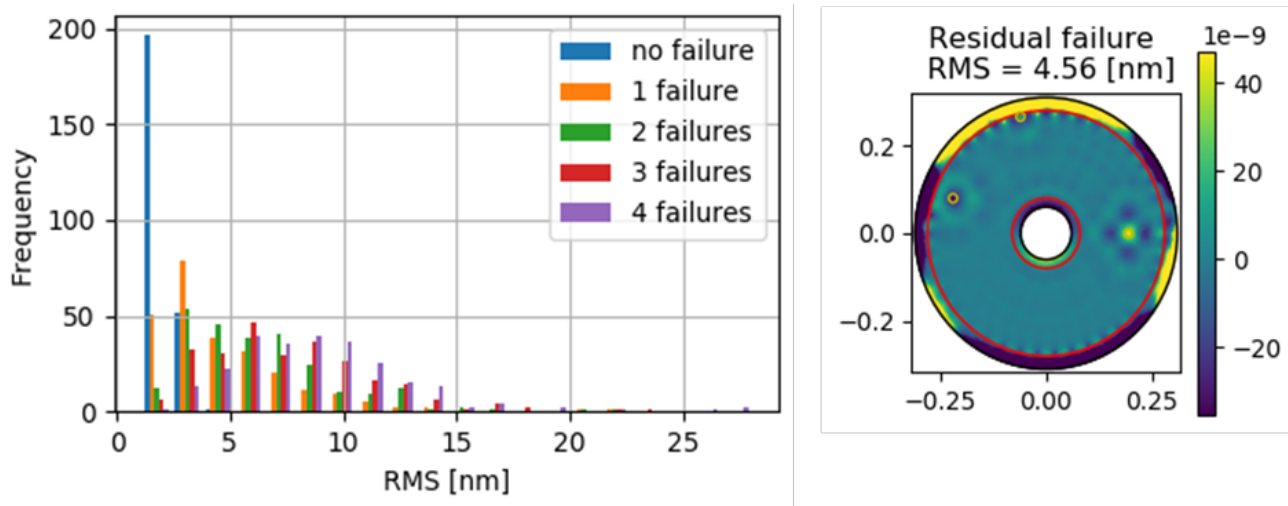

\begin{tabular}{|c|c|c|c|c|}
\hline \multicolumn{6}{|c|}{ Average RMS surface form error over 250 differen evaluations [nm] } \\
\hline no failures & 1 failure & 2 failures & 3 failures & 4 failures \\
\hline 2.03 & 4.60 & 6.18 & 7.52 & 8.77 \\
\hline
\end{tabular}

Figure 5: Actuator failure impact study results 


\section{DYNAMIC ANALYSIS}

Understanding the dynamical behavior of the ASM is important to achieve the required overall AO-control performance. The target disturbance rejection bandwidth is set at $100 \mathrm{~Hz}(-3 \mathrm{~dB})$ and is limited by the overall dynamic behavior of the ASM and the surrounding structure. This chapter discusses the dynamical FEM modeling results and the control strategy developed to control to cope with the ASM dynamics with the aim of meeting the $100 \mathrm{~Hz}$ bandwidth requirements.

\subsection{Dynamical FEM modeling}

Figure 6 shows the dominant resonance modes of the ASM obtained by dynamic FEM modeling. The first global resonance frequencies are relatively low, which requires special attention in the design of the mechanics and the feedback controllers. Since with this ASM concept the face sheet is rigidly supported via stiff actuators, the resonances of the mirror occur at relatively high frequencies $(>5 \mathrm{kHz})$.

For analysis of the achievable control performance and robustness, the main concerns are (i) the resonance modes that occur at a frequency close to the target control bandwidth, and (ii) mode shapes which show a significant displacement in the vertical direction as these might be excited by the actuator commands. As can be seen from Figure 6, these are mostly the tip and tilt resonances (\#7 and \#8) at 164Hz, and the astigmatism modes (\#43 and \#44) at $634 \mathrm{~Hz}$.

Dynamic behavior of the spider structure is also analyzed, showing that the low-frequency modes of the spider structure have little impact on the overall control performance of the ASM. This is explained by the fact that the modal mass corresponding to these resonance modes is significantly higher that the moving mass of the actuators.

\begin{tabular}{|c|c|l|}
\hline \# & Freq [Hz] & Description \\
\hline 7 & 164,28 & Tip Tilt \\
\hline 8 & 164,38 & Tip Tilt \\
\hline 9 & 182,26 & clocking \\
\hline 13 & 204,97 & piston \\
\hline 14 & 237,01 & clocking mirror against support \\
\hline 34 & 500,21 & ASM lateral translation \\
\hline 35 & 501,1 & ASM lateral mirror translation \\
\hline 43 & 634,84 & astigmatism \\
\hline 44 & 636,85 & astigmatism \\
\hline 45 & 679,46 & Lateral support piston/focus \\
\hline
\end{tabular}
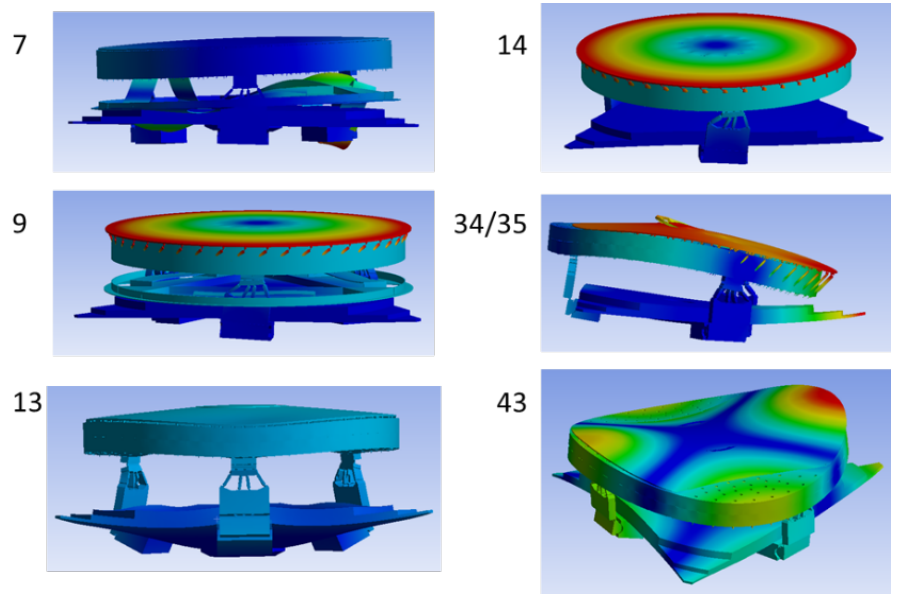

Figure 6: List of dominant dynamic modes (left), and a FEM image of their specific Zernike mode shapes

\subsection{Control strategy}

The resonances at frequencies near the target control bandwidth can be excited when the actuators are driven in a pattern that matches these dynamic mode shapes of the structure. Of particular concern are the tip/ tilt modes at $164 \mathrm{~Hz}$ and the astigmatism modes at $634 \mathrm{~Hz}$, as these have a significant deformation in the vertical direction, in-line with the actuation direction. The piston mode at $205 \mathrm{~Hz}$ is not considered, as the corresponding global piston commands are not of use in controlling the aberrations and thus will not be excited.

To handle these dynamics in the design of the feedback controller for the ASM, TNO is developing a control strategy based on modal-decoupling [5]. To study and explain the dynamic behavior of the ASM, a simplified dynamic model is initially used, of which the dynamic responses are shown in the bottom-left graph of Figure 7. To enable modal decoupling, the interaction matrices between the actuators and the wave-front sensors are decomposed to specific orthogonal patterns via the input and output matrices $T u$ and $T y$ in Figure 7. Commonly such a decomposition is based on Singular Value Decomposition (SVD) based on the set of measured interaction matrices between the actuators and wave-front sensor responses. However, in this case the first rows/columns of the input/output matrices are based on the specific mode shapes of the relevant low frequency modes (e.g. tip/tilt modes, and astigmatism modes). The remainder 
of the input/output matrices are based on SVD of the interaction matrices after subtraction of these dynamic mode shapes.

As a result, the excitation of the first resonance modes will only occur via those corresponding rows of the input matric $\mathrm{Tu}$, and are therefore decoupled from the rest of the system responses (see bottom-right graph in Figure 7). This enables robust control of the ASM via specifically tuned Single Input/Single Output (SISO) feedback controllers for each particular mode. As depicted in the bottom-right graph of Figure 7, the first rows of the decoupled dynamic system will show a typical fourth order response with a resonance and anti-resonance pair at the frequency of the corresponding resonance mode. Due to the decoupling, those resonances will not appear in the other system-responses which are thus not affected nor limited by these low frequency dynamics. The SISO-loops corresponding to the low frequency dynamic modes may be tuned to a lower feedback bandwidth to prevent instability from these modes, or more advanced control techniques may be applied such as notch-filters to push the bandwidth close to or beyond the frequency of these corresponding modes.

This effect of this control strategy has verified based on a dynamical FEM model of the UH 2.2 ASM. Figure 8 shows the achievable control bandwidth per mode in case of using standard Zernike decomposition, and when using the proposed combination of Modal-shapes and SVD decomposition. In the latter case the $100 \mathrm{~Hz}$ bandwidth requirement is met for all the mode apart from the first tip-tilt modes which are limited by the low tip-tilt resonances of $164 \mathrm{~Hz}$ (see Figure 6). Notice that this analysis assumes a standard decoupled Proportional-Integral (PI) feedback controller for each mode. It is expected that the bandwidth of the tip/tilt modes can be further improved utilizing more advanced feedback controller design for these modes, with for instance notch-filters to minimize the excitation of those specific modes.
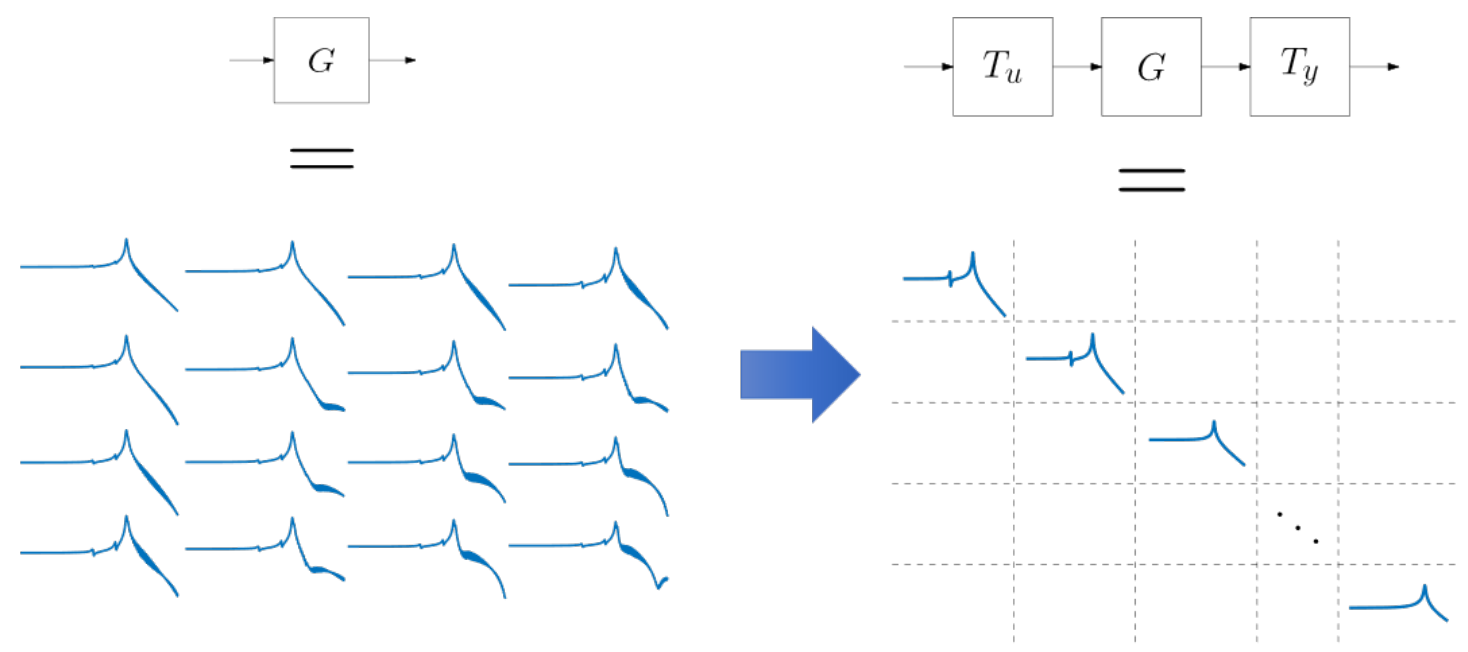

Figure 7: Principle of modal decoupling demonstrated via the simplified dynamical model. Input and output matrices are defined to diagonalize the ASM dynamic response 
Figure 8: Results of the dynamical analysis on the ASM, showing the achievable control bandwidth for each mode in case of Zernike decomposition (blue) and with a combination of modal and SVD decomposition (picture from [5]).

\section{OUTLOOK}

Based on the analysis discussed above, it is expected that the UH2.2 ASM will achieve is main performance goals in terms of Surface Form Error, and control bandwidths within its operational range. At the time of writing of this paper the main components of this ASM are being manufactured, and integration is scheduled for the beginning of 2021. After integration, its performance will be verified with initial functional tests, off-telescope tests at the summit, and finally full on sky-testing to demonstrate the expected imaging improvements. These tests are further described in [4].

In parallel to these activities, conceptual design studies are ongoing for application of this technology for an ASM for the APF-telescope, and the Keck-telescope [7].

\section{REFERENCES}

[1] Stefan Kuiper, Niek Doelman, Jet Human, Rudolf Saathof, Wimar Klop, Matthew Maniscalco, "Advances of TNO's electromagnetic deformable mirror development," Proc. SPIE 10706, Advances in Optical and Mechanical Technologies for Telescopes and Instrumentation III, 1070619 (10 July 2018); https://doi.org/10.1117/12.2311981

[2] Wouter Jonker, Stefan Kuiper, Jan Nijenhuis, Bert Dekker, Arjo Bos, Emiel van de Ven, Mark Chun, Hans Priem, Mathieu Breukers, "Design and MAIT Status of the UH2.2 Adaptive secondary Mirror," Proc. SPIE 11448, Astronomical telescopes and instrumentation, 1145120 (2020)

[3] Rachel Bowens-Rubin, Philip Hinz, Wouter Jonker, Stefan Kuiper, Cesar Laguna, Matthew Maniscalco, "Performance of Large Format-Deformable Mirror Constructed with TNO Variable-Reluctance Actuators," Proc. SPIE 11448, Adaptive Optics Systems VII, 11448234 (2020)

[4] Mark Chun, Christoph Baranec, Olivier Lai, Jessica Lu, Stefan Kuiper, Wouter Jonker, Matt Maniscalco, "A new adaptive secondary for astronomy on the University of Hawaii 2.2-meter telescope," Proc. SPIE 11448, Adaptive Optics Systems VII, 1144852 (2020)

[5] C.J.H. Langedijk, "Decentralized Control for Large Deformable Mirrors" Master-thesis report Technical University of Eindhoven, 2020

[6] Philip M. Hinz, Rachel Bowens-Rubin, Molly Kosiarek, Stefan Kuiper, Wouter Jonker, Matt Maniscalco, Mark Chun, Jessica Lu, Sam Ragland, "Developing adaptive secondary mirror concepts for the Shane and Keck Telescopes," Proc. SPIE 11448, Adaptive Optics Systems VII, 11448233 (2020) 